\title{
Dimensión espacial de las decisiones sobre manejo de tierras. Un modelo de análisis
}

\author{
The spatial dimension of decisions on land management. \\ An analysis model
}

\author{
Araceli del Carmen Andablo Reyes* (D) https://orcid.org/0000-0003-3940-0848 \\ María del Carmen Hernández Moreno** (D) https://orcid.org/0000-0002-8439-4232 \\ Jean Francois Mas *** (i) https://orcid.org/0000-0002-6138-9879
}

\section{Resumen}

En el debate actual sobre el cambio ambiental global y la contribución de las actividades humanas, como detonante de dicho proceso, se generan controversias disciplinarias. En este artículo se propone desarrollar un modelo de análisis para integrar la dimensión espacial en el estudio de las decisiones sobre manejo de tierras, y con ello superar las limitaciones de los trabajos que abordan la temática desde una perspectiva exclusivamente sociológica, y también de los que privilegian el abordaje espacial. En el modelo se conjuga el análisis de cambio de cubierta y uso de suelo con la teoría del actor-red. El resultado es un modelo híbrido, que le asigna a la dimensión espacial la categoría de elemento explicativo fundamental para comprender la toma de decisiones sobre manejo de tierras. La utilidad del modelo podría estar limitada por la disponibilidad de información a escalas geográficas detalladas, sin embargo aporta elementos para transitar de los modelos explicativos a los trasformadores de la realidad socionatural.

Palabras clave: toma de decisiones; manejo de tierras; teoría del actor-red; cambio de cubierta y uso de suelo; análisis espacial.

\begin{abstract}
In today's debate on global environmental change and contribution of human activities as a detonator of this process, disciplinary controversies are generated. This article aims to develop an analysis model in order to integrate the spatial dimension into the study of decisions on land management, and consequently overcome constraints of studies that deal with this subject from an exclusively sociological perspective, as well as those which privilege the spatial approach. In the mod$\mathrm{el}$, the cover change and land use analysis is combined with the actor-network theory. The result is a hybrid model that assigns the category of essential explanatory element to spatial dimension so as to understand decision making on land management. The usefulness of the model might be limited by the availability of information at detailed geographical scales; however, it provides elements for moving from the explanatory models to the transformers of socio-natural reality. Keywords: decision making; land management; actor-network theory; cover change and land use; spatial analysis.
\end{abstract}

Cómo citar:Andablo Reyes, A. C., Hernández Moreno, M.C., y Mas, J. F. (2019). Dimensión espacial de las decisiones sobre manejo de tierras. Un modelo de análisis. región y sociedad, 31, e977. doi: | 0.22 I98/rys20 I 9/3 |/977

* Autora para correspondencia. Centro de Investigación en Alimentación y Desarrollo A. C. Carretera a La Victoria, km 0.6, C. P. 83304. Hermosillo, Sonora, México. Correo electrónico: aandablo@ciad.mx

** Centro de Investigación en Alimentación y Desarrollo, A. C.

Carretera a La Victoria, km 0.6, C. P. 83304. Hermosillo, Sonora, México. Correo electrónico: mar@ciad.mx

*** Universidad Nacional Autónoma de México, campus Morelia, Centro de Investigaciones en Geografía Ambiental. Antigua carretera a Pátzcuaro \# 8701, colonia Ex-Hacienda de San José de La Huerta,

C.P.58190, Morelia, Michoacán, México.Correo electrónico:jfmas@ciga.unam.mx

Recibido: 29 de agosto de 2017

Reevaluado: 19 de febrero de 2018

Aceptado: 2 de abril de 2018

Liberado: 9 de enero de 2019 


\section{Introducción}

Entre el año 2000 y 2012 se estima que en el mundo se perdieron más de dos millones de $\mathrm{km}^{2}$ de bosque (Hansen et al., 2013, p. 850). Estos procesos de deforestación están asociados con el cambio ambiental global (CAG) que se experimenta desde hace algunas décadas. El Consejo Latinoamericano de Ciencias Sociales (CLACSO, 2018) define dicho concepto en tres sentidos: a) como paradigma de investigación, que abriga el conocimiento científico sobre el CAG (Castree, 2016); b) en términos del recuento de las trasformaciones negativas, tanto para los ecosistemas como para la vida humana (Grupo Intergubernamental de Expertos sobre el Cambio Climático [IPCC, por sus siglas en inglés], 2013, 2014) y c) como posibilidad de surgimiento de un paradigma nuevo que promueva el cambio social (O'Brien, 2012a, 2012b). El CAG registra y suma los cambios negativos en el planeta, asociados con las actividades humanas, que incluyen el climático, producto del aumento de las emisiones de gases atmosféricos de efecto invernadero (GEI); el rompimiento de la capa de ozono; la pérdida de biodiversidad; el cambio de usos de suelo y sus manifestaciones locales, como la escasez y la disminución de la calidad del agua, y la pérdida de la fertilidad de los suelos (Leichenko y Eisenhauer, 2017). Según el IPCC, el calentamiento observado desde mediados del siglo XX es inducido por la influencia humana (2013, p. 17), y estimó que en 2010 del total de emisiones de los GEI en el mundo, 24\% fueron generadas por la agricultura, el cambio de uso de suelo y las actividades forestales (2014, p. 44).

La toma de conciencia sobre los límites que estos cambios le están provocando al modelo de desarrollo económico, impuesto a partir de la segunda guerra mundial, ha detonado el surgimiento de diversos enfoques desde las ciencias sociales, con el fin de orientar las acciones sobre sus consecuencias ambientales, y proponer estrategias para revertir o mitigar sus efectos. Sin embargo, estos esfuerzos han sido insuficientes, y no se han traducido en acciones efectivas que influyan sobre la toma de decisiones de actores políticos, empresariales o sociales (Aranda, 2004; Castree, 2016). El IPCC (2013, p. 20) estima que entre 2016 y 2035, es posible que la temperatura media global pueda superar el rango de 0.3 ${ }^{\circ} \mathrm{C}$ a $0.7^{\circ} \mathrm{C}$, con respecto a la registrada en el periodo 1986-2005.

En este artículo se plantea que los enfoques sociológicos presentan límites ontológicos, epistemológicos y, por ende, metodológicos, para abordar la problemática ambiental y responder la pregunta central que constituye el punto de partida en esta tarea: ¿cómo se toman las decisiones sobre el manejo de tierras?, es decir, ¿qué entidades y qué tipo de relaciones impulsan a los tomadores de decisiones sobre el uso de sus tierras? Estas limitaciones se originan, por una parte, en el debate histórico sobre los determinantes de la acción social, que constriñen la respuesta a esta pregunta entre los extremos de la conducta racional y calculadora de los individuos para lograr la máxima ganancia, y las restricciones que les imponen las estructuras sociales a los tomadores de decisiones (Williams y Patterson, 1996, p. 516). Por otra parte, en este acercamiento a la cuestión ambiental, en el campo de los estudios rurales, los enfoques sociológicos han caído en la trampa de origen ontológico, que plantea la separación dualista entre sociedad y naturaleza (Goodman, 1999). Una consecuencia epis- 
temológica y metodológica derivada de lo anterior, es la débil integración de la dimensión espacial como elemento determinante en el análisis de las decisiones sobre manejo de tierras, sin duda imprescindible en los estudios ambientales, porque la deforestación así como otros detonantes del CAG, están inscritos sobre la superficie terrestre.

Las decisiones sobre el manejo de tierras dejan una huella espacial histórica, a la que se le ha dado seguimiento a través del análisis de los cambios de cubierta y uso de suelo (CCUS) aprovechando los avances de la percepción remota y los sistemas de información geográfica. El uso de suelo se refiere al aprovechamiento humano de la tierra como recurso, estudiado ampliamente por las ciencias sociales; la cubierta de suelo denota el carácter físico y biótico de la superficie terrestre, abordada de manera profusa por las ciencias naturales (Meyer y Turner, 1992, p. 39).

El análisis de CCUS se sirve de los marcos conceptuales de las ciencias sociales para encontrar explicaciones a los procesos. No obstante requiere ajustar conceptos, variables e indicadores sociales a una dimensión espacial, y trabajar en un ambiente de rigurosidad estadística. En esta adaptación, si bien ganan confiabilidad estadística, la pierden en términos de su capacidad de representar el carácter multifactorial y dinámico de los fenómenos sociales, ya que no todas las variables de este tipo se pueden expresar de forma espacial, ni en escalas diferentes.

El objetivo del presente artículo es desarrollar un modelo de análisis para integrar la dimensión espacial en el estudio de las decisiones sobre manejo de tierras, y vincular algunos elementos de la teoría del actor-red (TAR) y del análisis espacial de los CCUS. En principio se sitúan los elementos conceptuales principales involucrados en el debate sobre el CAG; luego se discute la forma en que algunos enfoques sociológicos han abordado el tema, incorporan a la naturaleza e integran la dimensión espacial; más adelante se revisan los alcances y limitaciones del análisis de CCUS. Al final se plantea cómo la TAR, a través de algunas premisas, evade los dualismos conceptuales agencia-estructura y sociedad-naturaleza y cómo, según estas premisas, se puede establecer un vínculo teórico-metodológico entre el análisis de CCUS y la TAR, para rastrear las asociaciones espaciales producto de las redes que se tejen en torno al manejo de tierras. El planteamiento metodológico final resulta un híbrido que integra la dimensión espacial en la explicación de la toma de decisiones, y supera algunas limitaciones del análisis de CCUS, a través del seguimiento de las relaciones espaciales en las interacciones locales.

\section{Elementos en controversia sobre el cambio ambiental global}

Para iniciar es necesario situar algunos elementos conceptuales en la controversia que se trata de abordar, primero se debe partir de lo que aquí se entenderá por controversia: "situaciones donde los actores están en desacuerdo" (Venturini, 2010, p. 261). Puede parecer poco relevante definir esta palabra, sin embar- 
go, una controversia o un desacuerdo siempre es el mar donde se navega en el planteamiento de cualquier problema, constituye el estado de cosas de donde se jalan los hilos que se van a seguir y, más que eso, es el punto de partida donde se sitúa el investigador. Entre más compleja es la controversia, más fácil es observar la diversidad de actores que participan de ella. En este sentido, la del cambio ambiental global es un caldo de cultivo para el desarrollo de cualquier investigación, es decir, que no tiene límites disciplinarios.

Un primer elemento que define la controversia sobre el CAG es su carácter multidisciplinario, sobre todo, la necesidad metodológica de su abordaje interdisciplinario; en segundo lugar, destaca que en ella participan actores humanos y no humanos, una premisa de la TAR que se profundizará más adelante y, en tercero, que la participación en ella constituye una necesidad para los humanos, - apenas reconocida a fines de siglo pasado (Giddens, 2014)-, debido a que el CAG representa una amenaza para su subsistencia (DellaSala et al., 2018).

De la multidisciplinariedad se deriva que uno de los conceptos principales involucrados es el CCUS, una categoría híbrida que registra la huella espacial que genera el manejo de tierras que, por un lado, se refiere al estudio de las actividades humanas que alteran directamente el ambiente físico pero, a la vez, registra cómo los cambios que éstas generan sobre la superficie terrestre tienen consecuencias ambientales que, en última instancia, inciden en el uso de suelo (Meyer y Turner, 1992).

Desde la perspectiva espacial, los estudios de CCUS se han concentrado en resaltar los efectos que la actividad humana genera sobre las cubiertas de suelo, sin profundizar sobre la reorganización espacial que estos cambios producen en ella. Sin embargo, este último efecto se ha identificado con claridad desde perspectivas economicistas, de ahí la tercera característica que involucra la subsistencia humana. Las pérdidas de grandes extensiones de cultivos agrícolas por heladas, sequías o inundaciones, que luego se traducen en crisis alimentarias globales, incremento de pobreza y de migraciones en condiciones precarias han dado lugar a propuestas continuas de los organismos internacionales que plantean, al menos en el discurso, la necesidad de modificar la forma en que el ser humano se relaciona con la naturaleza.

Así se introduce en el debate el concepto manejo de tierras. El Banco Mundial (BM) se refiere al manejo sostenible de la tierra como el procedimiento que integra también el del agua, la biodiversidad y el medio ambiente, para satisfacer necesidades preservando los ecosistemas y las formas de subsistencia (BM, 2008, p. 2). La Food and Agriculture Organization (FAO, 2016) señala que el manejo de tierras implica todas las prácticas para la protección y mejoramiento de los bienes y servicios proporcionados por la tierra. Desde esta perspectiva, el concepto se acerca a lo que Meyer y Turner (1992) definen como uso de suelo, en términos del aprovechamiento humano de la tierra como recurso; se aborda más bien desde la planeación del desarrollo. Antes de la visión instrumental del concepto está el debate sociológico sobre cómo se deciden esos procedimientos y prácticas que se aplican en las tierras, en otras palabras, cómo se toman las decisiones sobre el manejo de tierras, sobre el que se profundiza en el apartado que aborda el dualismo agencia-estructura. 
Los conceptos instrumentales del BM y la FAO pretenden introducir una perspectiva de preservación, sin embargo, conciben a la tierra como sinónimo de recurso, porque al final estos organismos se basan en una concepción modernista de la relación sociedad-naturaleza, donde el ser humano posee control sobre las entidades naturales (Rosales, 2006); la misma visión que ha impuesto límites ontológicos, epistemológicos y metodológicos al abordaje de la problemática ambiental (Mastrangelo, 2009). Para tomar distancia de esta visión de dominio y de apropiación, aquí se eligió abordar el tema de manejo de tierras y no de recursos naturales o del territorio. En este sentido, la tierra se reconoce en su dimensión más compleja, como sinónimo de campo de vida o biosfera, según lo planteado por Vernadsky (1944, citado en Huggett 1999, 426), para quien "la biosfera era todos los sistemas de vida y de soporte de vida -los organismos vivos y el medio en el que viven- (aire, agua, suelo, sedimentos)" y, por ende, el manejo de tierras se define como un proceso de negociación entre los tomadores de decisiones y sus tierras, desarrollado a través de una red de mediadores humanos y no humanos, que amplían o limitan las oportunidades de toma de decisiones (Gray y Gibson, 2013).

\section{Abordajes sociológicos sobre las decisiones de manejo de tierras}

El debate sobre la acción social y económica ha transitado entre los extremos planteados por la perspectiva individualista, donde la conducta racional del individuo es la que conduce a la toma de decisiones (Smelser y Swedberg, 2005); y el funcionalismo estructural de Durkheim, cuyo postulado básico es que el individuo actúa con base en reglas, normas y valores generados en sociedad (Mariñez, 2005). Sin embargo, en la evolución del pensamiento sociológico la integración del espacio es reciente porque mientras el paradigma positivista fue dominante, el conocimiento tenía una connotación universal que no admitía cuestionamiento espacio-temporal (Raedeke y Rikoon, 1997).

En un estudio de caso, Raedeke y Rikoon (1997) analizaron el tipo de conocimientos que emplean los agricultores para decidir sobre la introducción de un fertilizante nuevo, considerando la reorganización del espacio que implicaría. En este enfoque, centrado en las decisiones individuales, el actor parece estar libre de presiones estructurales. Sin embargo, este producto nuevo no llega libre de carga "estructural", forma parte de una cadena agroindustrial, es probable que lo introduzcan técnicos agropecuarios que trabajan para dependencias gubernamentales, o las propias agroindustrias, quizá también viene acompañado de un programa de estímulos para su introducción, entre otras circunstancias que inciden sobre las preferencias del agricultor por un conocimiento u otro. Williams y Patterson señalan que las perspectivas individualistas, entre ellas la psicología ambiental, se concentran en "estudiar los significados como fenómenos a nivel individual expresados a través de motivaciones, experiencias, beneficios, satisfacciones y metas, prestando poca atención a cómo estos significados están socialmente estructurados" (1996, p. 516). En el mismo sentido, Cote y 
Nightingale señalan respecto a la toma de decisiones en un caso semejante al de Raedeke y Rikoon (1997), que "el conocimiento colisiona con las estructuras de poder cuando tratamos de entender 'la manera correcta de hacer las cosas'” (2012, p. 483).

Desde algunas perspectivas sociológicas, las relaciones de poder son el elemento clave que vincula las decisiones individuales con los contextos en que está inmerso el actor. A través de la noción de campo, capital y habitus, Pierre Bourdieu propone una forma de superar el debate agencia-estructura en la determinación de la acción social. "En términos analíticos, un campo puede ser definido como una red o una configuración de relaciones objetivas entre posiciones" (Bourdieu y Wacquant, 2005, p. 150). Estas posiciones son ocupadas por agentes o instituciones, y definidas por sus posibilidades de acceso a "especies del poder (o capital) cuya posesión ordena el acceso a ventajas específicas que están en juego en el campo, así como por su relación objetiva con otras posiciones ([de] dominación, subordinación, homología, etcétera)” (Bourdieu y Wacquant, 2005, p. 150).

Los campos sociales se pueden comparar con espacios de juego y competencia, donde los jugadores, agentes o instituciones disponen de capital económico, cultural, social, simbólico y político (Eichholz, Assche, Oberkircher y Hornidge, 2012, p. 9), para realizar sus movimientos (tomar decisiones), que los jugadores orientan a través del habitus, "conjunto de actitudes y creencias internalizadas que son desarrolladas al vivir y socializar en un ambiente específico" (Bourdieu, 1997; citado en Eichholz et al., 2012, p. 874). El habitus es producto de la experiencia adquirida por los actores en los campos donde se desenvuelven, donde toman posiciones, es una negociación entre la rigidez de la estructura social, configurada en los campos, y la interpretación individual.

Eichholz et al. (2012) utilizan este planteamiento complejo para entender la competencia sobre el acceso a la tierra y al agua en una economía rural, donde los actores luchan por mejorar sus posiciones en el campo económico. La localización de las tierras constituye un elemento que las capitaliza, con base en tres condiciones espaciales: calidad del suelo, elevación y distancia hacia el canal principal de riego. También les confieren valores a las tierras de cultivo de los agricultores, pues forman parte de su capital económico y determinan su posición en la economía rural. Ellos establecen relaciones múltiples con la tierra: obligaciones, expectativas, derechos, riesgos, deudas y ganancias (Eichholz et al., 2012, p. 21), todas acotadas por su papel como recurso, como capital económico.

Como se confirma en el estudio de caso, las posiciones espaciales en Bourdieu son relevantes en tanto que pertenecen al espacio social. La localización de la tierra, así como todas sus cualidades, tiene sentido solo en función de otorgar posición a los agentes, a través de su relación de posesión o de propiedad (Bourdieu, 1999). El papel de las cosas, entre ellas la tierra, en este orden teórico tiene un trasfondo ontológico, que es importante destacar: el actor posee las cosas, se las apropia y las carga como fichas de juego (Bourdieu y Wacquant, 2005, pp. 152-153), para conservar, mejorar o perder su posición en el campo de juego o espacio social. 
La teoría de la estructuración de Giddens es otra perspectiva de la acción social, ubicada en este intento de superación del dualismo agencia-estructura. Si bien establece que el objetivo básico de las ciencias sociales es el estudio de "las prácticas sociales ordenadas a través del espacio y el tiempo" (1984, p. 2); define la estructura como "un conjunto de reglas y recursos organizado de forma recurrente [...]", que es estable e independiente del tiempo y el espacio (1984, p. 25); aunque al mismo tiempo argumenta una condición de dualidad de la estructura porque "[...] las propiedades estructurales de los sistemas sociales son tanto medio como resultado de las prácticas que ellos [los agentes] recurrentemente organizan" (1984, p. 25). En este sentido, se puede decir que existe una relación dialéctica entre la práctica de los actores y la constitución de la estructura.

Es imposible sintetizar en unas líneas la producción teórica de Bourdieu y Giddens, no obstante aquí lo importante es destacar que, si bien el espacio es un elemento integrado en el cuerpo teórico de ambos enfoques, se restringe al social, dialécticamente construido en la estructuración o bien definido mediante la dinámica de las posiciones en los campos. El espacio físico, en específico el geográfico, constituye un elemento de contexto y en este mismo orden epistemológico se entiende la relación humano-naturaleza, como una de dominación no de interacción, en tanto la categoría de actor está restringida a los humanos.

\section{El dualismo naturaleza-sociedad y el lugar de lo espacial}

En un esfuerzo de integración entre la ciencia física y la social, uno de los principales marcos analíticos sobre el CAG y la forma de enfrentarlo se ha desarrollado desde la teoría de sistemas. El concepto de sistemas socioecológicos (SES) reconoce la integralidad de uno que incluye las relaciones humano-ambientales (Berkes, Colding y Folke, 2003). Resiliencia, vulnerabilidad y capacidad adaptativa son conceptos clave en el análisis de la respuesta de los SES, frente a estresores como la globalización y el cambio climático (Young et al., 2006). La dimensión espacial en este enfoque es central, porque observa principalmente el comportamiento del sistema ante la ocurrencia de amenazas físicas, como eventos climáticos extremos.

La ecología adoptó el concepto de resiliencia para darle un giro social, y definirla como "la capacidad de grupos o comunidades para hacer frente a tensiones y disturbios externos como resultado de cambios sociales, políticos y ambientales" (Adger, 2000b, p. 347; citado en Cote y Nightingale, 2012, p. 478). La resiliencia se presenta como una propiedad emergente del sistema, que permite absorber cierta cantidad de cambio sin alterar las relaciones y funciones del SES (Cote y Nightingale, 2012; Berkes et al., 2003, p. 6), y se considera deseable para los SES. Sin embargo, esta interpretación constituye la debilidad principal del enfoque, porque oculta la importancia de las relaciones de poder que sostienen el equilibrio de un sistema y que pueden, por lo tanto, constituir los factores resilientes más importantes, pero no deseables (Cote y Nightingale, 2012), des- 
estima el análisis histórico de la construcción de la vulnerabilidad de un SES, y obvia que una respuesta resiliente del subsistema ecológico no necesariamente se traduce en una positiva para el subsistema social o viceversa.

Desde esta perspectiva sistémica se han generado las mayores aportaciones de los geógrafos a la investigación sobre el CAG. Sin embargo, además de las críticas al marco analítico señaladas, se han sumado otras que apuntan hacia las dificultades de traducir estos marcos analíticos en acciones efectivas para revertir el CAG. O’Brien (2012a) señala que estos enfoques se han centrado más en buscar alternativas de adaptación al CAG, como si ya no fuera posible trabajar en su trasformación, incluso se pregunta si están contribuyendo a perpetuar los paradigmas que genera el CAG (O'Brien, 2012b, p. 593).

En el debate por integrar a la naturaleza en el análisis sociológico, han surgido enfoques interdisciplinarios, como la sociología ambiental, que tratan de abordar el estudio de esta relación de manera integral (Aranda, 2004). Sin embargo, solo han conseguido teorizar a la naturaleza "en términos externalizados y mecanicista, y por lo tanto abstraída del ámbito social” (Goodman, 1999, p. 17). Esta visión se encuentra enclavada en el propio origen de la ciencia, en la forma en que se apartan las ciencias sociales de las físicas y, por tanto, influye en cómo se integran ambos grupos disciplinarios en la búsqueda de alternativas a la problemática del medio ambiente. Aranda (2004, p. 206) señala que a pesar de los desarrollos teóricos de la sociología ambiental, aún existe un vacío en la integración de la racionalidad ambiental, cuando se trata de proponer soluciones al problema, mientras que Williams y Patterson (1996, p. 516) afirman que "el desafío para los científicos naturales y sociales es identificar formas de trascender la naturaleza fragmentaria de la investigación científica para informar sobre la gestión de los ecosistemas."

En este artículo se argumenta que estas debilidades disciplinarias se derivan de una posición ontológica del ser humano frente a la naturaleza: “El hombre moderno no está en la naturaleza porque está ante ella [...]" (Mendiola, 2012, p. 245) comprendiendo sus procesos, para extraer el máximo beneficio de ella. La tarea de las ciencias físicas ha sido reproducir la ilusión del control mediante desarrollos tecnológicos: fertilizantes, plaguicidas, organismos genéticamente modificados y maquinaria, entre muchos. Desde esta posición de dominio y, por tanto, de independencia respecto al entorno ambiental, la dimensión espacial pierde relevancia epistemológica y metodológica en el estudio de las decisiones sobre manejo de tierras. El espacio deja de ser un elemento explicativo, para constituirse en uno descriptivo del lugar donde suceden los procesos sociales (Moreno, 1988). El espacio solo se puede explicar socialmente, porque se produce socialmente, en una suerte de relación unilateral.

Karl Polanyi (2007), en 1947, en su crítica del liberalismo económico reflexionaba sobre la soberbia del hombre respecto a la posición que le asignaba a la tierra en el sistema naciente entonces:

[...] la empresa más extraña de todas las emprendidas por nuestros antepasados consistió quizás en aislar a la tierra y hacer de ella un mercado. [...] Nosotros podríamos imaginarnos con dificultad a un hombre que viene al mundo sin brazos ni piernas, o, lo que es parecido, a un hombre 
que arrastra su vida sin tierra. Sin embargo, separar la tierra del hombre y organizar la sociedad con el fin de que satisfaga las exigencias de un mercado inmobiliario, ha constituido una parte vital de la concepción utópica de una economía de mercado (pp. 291-292).

Mastrangelo (2009, p. 342) relaciona esta idea externalizada sobre la naturaleza con la influencia de "la comprensión teológica cristiana del Universo". La revisión que realiza Valembois (1981) sobre la relación humano-naturaleza en el indio mesoamericano refuerza este argumento; compara la filosofía sobre la naturaleza en el Popol Vuh y la visión cristiana del mundo y la creación: "En el Popol Vuh, los mismos dioses son seres naturales o seres de la naturaleza; en cambio en la tradición occidental el Dios está por encima de ella" (1981, p. 116). En su argumento describe cómo los indios mesoamericanos fueron despojados de su vínculo con la tierra, a través del repartimiento y la encomienda: "para el indígena era un contrasentido hablar de tenencia de la tierra, con su terminología económicamente connotada de compra y venta [porque] la tierra no pertenece al hombre, el hombre pertenece a la tierra” (1981, p. 117).

\section{Sustituir dualismos por relaciones simétricas}

En este trabajo no se pretende importar la visión ontológica de los antepasados al mundo de las ciencias sociales, pero sí retomar de esta filosofía el reconocimiento de la capacidad de interacción de la tierra. Por esta razón se optó por considerar a la tierra como sinónimo de biosfera, como una red en donde el ser humano es uno de los partícipes, a través de interacciones múltiples.

A pesar de que en el medio rural las mediaciones entre el ser humano y la tierra también han experimentado la influencia de la tecnología, sigue siendo un espacio privilegiado para observar estas interacciones, porque aún es posible que en la vida cotidiana se perciba el poder de la tierra y sus componentes sobre las decisiones de manejo. Sin duda, los avances tecnológicos y la industrialización han contribuido a expandir la brecha ontológica, y mediar las relaciones entre el ser humano y la tierra, a través de la tenencia de ésta, las maquinarias, las semillas mejoradas, los fertilizantes y los plaguicidas.

En efecto, la tecnología junto con los programas de fomento, los subsidios y los técnicos agropecuarios, entre otros elementos, actúan como mediadores entre los tomadores de decisiones y sus tierras. Sin embargo, estas mediaciones no son unidireccionales, la tierra también responde a las acciones humanas con suelos erosionados, resistencia de plagas, sequías recurrentes, inundaciones y todos los síntomas del cambio ambiental estudiados.

Según la TAR, "cualquier cosa que modifica con su incidencia un estado de cosas, es un actor" (Latour, 2008, p. 106). El hecho de que el ser humano tenga que modificar sus conductas ante los problemas ambientales le otorga a la tierra esta cualidad. Latour señala que "[...] las capacidades sociales básicas aportan solo un diminuto subconjunto de las asociaciones que componen las sociedades" (2008, p. 103), el resto proviene de las relaciones establecidas con objetos que participan de la acción. 
El principio de simetría en la TAR implica reconocer que los elementos no humanos, "las cosas que interactúan con los humanos" (Latour, 2008, p. 107), tienen capacidad de influir en otros actores. Este principio permite exponer el papel que juegan los elementos no humanos, que intervienen en la configuración de las redes que se tejen en torno al manejo de tierras, y comprender cómo dichas redes están ancladas y definidas espacialmente. La TAR es una forma de abordaje metodológico que puede integrar al espacio como un elemento explicativo, porque la dimensión espacial constituye un atributo de los actores, en particular de la tierra, que condiciona la toma de decisiones.

\section{Teoría del actor-red}

“Un 'actor', tal como aparece en la expresión unida por un guion actor-red, no es la fuente de una acción sino el blanco móvil de una enorme cantidad de entidades que convergen hacia él” (Latour, 2008, p. 73). Estas entidades son otros actores-red que actúan como mediadores, "transforman, traducen, distorsionan y modifican el significado o los elementos que se supone que deben transportar" (Latour, 2008, p. 63). El desarrollo de la acción es visible en esta cadena de mediaciones entre actores-red, incluidas las cosas aunque, como señala Latour (2008):

La TAR no es la afirmación vacía de que son los objetos los que hacen las cosas "en lugar de" los actores humanos: dice simplemente que ninguna ciencia de lo social puede iniciarse siquiera si no se explora primero la cuestión de quién y qué participa en la acción, aunque signifique permitir que se incorporen elementos que, a falta de mejor término, podríamos llamar no-humanos (p. 107).

Para responder la pregunta formulada en este artículo, trabajar según los principios de la TAR significa rastrear las entidades que convergen hacia los tomadores de decisiones, desplegar las relaciones y los actores-red que participan en esta toma de decisiones. Estas relaciones no son neutrales "transportan autonomía o esclavización [...]" (Latour, 2008, p. 309), y se fortalecen o debilitan mediante la participación de los mediadores no humanos.

La formación de una red se puede describir como un proceso de traducción que implica varios momentos en el comportamiento de los actores: problematización, interesamiento, enrolamiento y movilización (Gray y Gibson, 2013). El primero incluye el planteamiento o la existencia de un interés compartido por diversos actores, por ejemplo, en un caso de decisiones sobre manejo de tierras, este interés podría ser el incremento de la productividad agropecuaria que conlleva al aumento de los ingresos de los productores rurales. El interesamiento sucede cuando ese interés primario, que puso en contacto a los actores, se fortalece tanto que define roles de acción. En este momento la importancia de los mediadores no humanos es crucial: planes de desarrollo, programas de financiamiento, semillas mejoradas, suelos, cuerpos de agua y tecnologías, así como otros elementos decisivos en la definición de estos roles. Cada actor-red 
será estimulado por otros para hacer cosas, si el tomador de decisiones se enrola, entonces decidirá contratar un crédito, aplicar un fertilizante o comprar implementos agrícolas nuevos; una vez que se fortalece el interés y se definen los roles se produce un enrolamiento, cada actor asume su rol como el mejor posible para lograr sus objetivos, se establecen rutinas de participación. Por último se genera la movilización, donde las redes tienen cierta estabilidad, en tanto los intereses compartidos mantengan enrolados a los participantes y éstos continúen cumpliendo su rol (Gray y Gibson, 2013): el fertilizante incremente el rendimiento por hectárea, el crédito llegue, el suelo conserve sus nutrientes y el tomador de decisiones obtenga lo que necesita para sobrevivir o acumular, cualquiera que sea su lógica de producción.

El seguimiento del proceso de traducción, en el estudio de la toma de decisiones sobre manejo, permite identificar las motivaciones que cada participante de la red tiene para mantenerse enrolado, y actuar de cierta forma. Gray y Gibson (2013) realizaron un estudio desde esta perspectiva, para entender cómo un grupo de agricultores se enroló en una cadena agroindustrial con prácticas productivas insustentables. Los resultados indican que a través de diversos mecanismos como incentivos financieros, créditos y precios altos, los agricultores quedaron enrolados de forma subordinada en un ciclo de degradación que introdujo a mediadores nuevos entre ellos y sus tierras (Gray y Gibson, 2013, p. 87). Este rol de subordinación se consolidó en el proceso de traducción o de formación de la red, cuando pocos actores "obtienen el derecho a expresar y representar a los muchos actores silenciosos de los mundos sociales y naturales que han movilizado" (Callon, 1986; citado en Gray y Gibson, 2013, p. 95).

Una representación simple, pero precisa, de las redes es la forma de estrella: puntos donde nacen o donde llegan líneas que conectan con otros puntos igualmente ramificados (Latour, 2008). El seguimiento y descripción de estas interacciones es suficiente para encontrar las explicaciones de la acción, no es necesario hacer referencia a cuestiones de contexto, a los procesos estructurales o al nivel macro, para explicar las acciones locales o micro. Desde la perspectiva de la TAR, pasar de lo micro a lo macro requiere establecer una interacción, trazar una línea más en la estrella. La estructura, lo macro, o bien el contexto está contenido en las interacciones locales. "La naturaleza circulante de lo social significa que cada interacción local es un efecto de red, y cada red

es una 'recapitulación' de actividades locales” (Latour, 1999; citado en Gray y Gibson, 2013, p. 84). Lo macro solo constituye una red más extensa y compleja, son procesos de traducción que involucran a muchos actores, pero que también son observables en sus interacciones locales (Latour, 2008).

\section{Análisis sobre el CCUS}

La cubierta de suelo se define como "los atributos de la superficie terrestre y la sub-superficie inmediata, incluyendo la biota, suelo, topografía, aguas superficiales y subterráneas, y las estructuras humanas" (Lambin, Geist y Lepers, 2003, p. 213). Estas cubiertas de suelo son clasificadas en unidades espaciales definidas por ciertos atributos biofísicos y/o sociales, como tipo de vegetación y uso 
de suelo. De esta forma, un mapa de cambio se construye con las categorías que presentan un cambio de atributos de una fecha a otra, donde los insumos básicos de dicha clasificación son principalmente imágenes satelitales.

El análisis de CCUS se propone identificar los conductores del cambio, con este término se denomina a las variables que se pueden manipular en una base de datos referida espacialmente. Para ello se construyen modelos de cambio mediante la asociación de variables que se agrupan como de origen físico o social, para identificar los efectos que tienen sobre la distribución espacial de los CCUS. Por lo general, estos cambios son producto de la deforestación, debido a la ampliación de la frontera agrícola, pecuaria, urbana y/o forestal. Para establecer algunas hipótesis sobre el origen del cambio, las variables se analizan a partir de procesos sociales de gobernanza, demográficos o de la influencia de las políticas públicas sobre las decisiones de cambios en el uso de suelo. La integración de estos elementos dinámicos al modelo también permite generar escenarios prospectivos, para visualizar las hipótesis sobre el comportamiento de los conductores del cambio a futuro (Chowdhury, 2006; Geist y Lambin, 2002; Soares et al., 2004).

El análisis sobre CCUS consiste en construir modelos estadísticos espaciales de diversa complejidad, entre los más sencillos están los econométricos (National Research Council [NRC], 2013). Éstos relacionan los conductores del cambio o bien las variables explicativas del modelo con los CCUS generados en un periodo específico, los cuales representan la variable dependiente por modelar. Los conductores del cambio se clasifican en causas inmediatas o directas sobre el CCUS, como la expansión de la frontera agrícola, la extracción de madera y la ampliación de la mancha urbana; y causas subyacentes o de raíz, que son las fuerzas fundamentales que sustentan a las primeras y se agrupan en factores demográficos, económicos, tecnológicos, culturales, políticos, institucionales y biofísicos (Geist y Lambin, 2002, p. 144; Lambin et al., 2003, p. 216). Estas causas subyacentes pueden promover o inhibir la deforestación. En el primer caso se pueden agrupar políticas públicas de fomento de las actividades agropecuarias, apertura de mercados o expansión de los existentes; y, en el segundo, iniciativas de conservación por parte de organismos gubernamentales o no gubernamentales, pagos por servicios ambientales o abandono de tierras de cultivo por migración.

Las variables biofísicas que se integran al modelo representan elementos importantes en el desarrollo de las actividades humanas. Por ejemplo, los sitios con pendientes planas o menos pronunciadas se consideran aptos para establecer usos urbanos, agrícolas o pecuarios; así como las superficies con mayor precipitación son más aptas para los cultivos, sobre todo de temporal. Estos supuestos, sobre las preferencias de uso en relación con aspectos biofísicos del espacio, se basan en la teoría de la renta de la tierra de Ricardo (Chowdhury, 2006; Geoghegan et al., 2001), que señala que los agricultores seleccionan las nuevas tierras de cultivo en función de su fertilidad, por lo tanto las áreas con mayor precipitación y suelos más fértiles tendrán mayor probabilidad de ser deforestadas.

La localización es un factor importante en los estudios de CCUS, porque se espera que el riesgo de deforestación se incremente por la facilidad de acceso 
a los mercados, a las carreteras, a las tierras agrícolas ya existentes y a los cuerpos de agua (Chowdhury, 2006; Serneels y Lambin, 2001). Por lo general, los factores de proximidad están muy correlacionados con la deforestación, lo que hace evidente la importancia de las relaciones espaciales en la toma de decisiones sobre el uso del suelo.

Los modelos espaciales de análisis de CCUS se someten a pruebas de rigurosidad estadística, para garantizar su fiabilidad. Sin embargo, algunos autores reconocen las limitaciones de este enfoque, y señalan que es necesario mejorar los modelos con información generada en campo (Overmars y Vergurg, 2005; Vance y Geoghegan, 2002). Entwisle, Walsh, Rindfuss y Chamratrithirong estudiaron la relación entre los procesos migratorios y los cambios de uso de suelo, con base en la generación de información histórica y referida espacialmente sobre las dinámicas de población en una región de Tailandia, y concluyeron que las imágenes satelitales ofrecen "una valiosa perspectiva del paisaje que se ve reforzada sustancialmente a través de la disponibilidad de los datos sociales correspondientes" (1998, p. 140).

\section{Desajustes temporales y espaciales de los conductores del CCUS}

Con el fin de representar las relaciones sociales, económicas e institucionales asociadas con los CCUS, se utilizan variables construidas con estadísticas oficiales sobre producción, consumo y oferta gubernamental, que no están georeferidas y cuya temporalidad es limitada. Esta condición provoca que los ejercicios de modelación traten de relacionar procesos de CCUS ocurridos en un periodo que no coincide de manera adecuada con la temporalidad de las variables explicativas. Por ejemplo, Chowdhury (2006) trata de explicar un fenómeno de cambio de uso de suelo que sucedió entre 1987 y 1996, con estadísticas de población de 1990 y con la inversión total realizada en la zona de estudio entre 1990 y 1999. Es evidente que estos desajustes temporales limitan los resultados de la modelación (Chowdhury, 2006; Pineda, Bosque, Gómez y Franco, 2010; Serneels y Lambin, 2001).

El análisis espacial implica que las variables explicativas, así como los CCUS, serán mapeados en una unidad espacial que constituye un polígono, al que se asocian los atributos de los conductores del cambio y de la variable dependiente que representa los CCUS. En el caso de éstos, la unidad espacial es más flexible porque pueden ser representados casi de forma continua sobre el espacio, al igual que las variables físicas, como los datos de temperatura y precipitación. Sin embargo, las variables sociales por lo común se encuentran disponibles a escalas geográficas agregadas en unidades político-administrativas, como los municipios o entidades federativas, y esto tiene consecuencias en la interpretación de los resultados, porque se pierden los fenómenos que suceden a escalas más finas, como las localidades, o con una distribución espacial distinta, como el tipo de tenencia de la tierra. 
En el análisis espacial esta complicación se ha estudiado a profundidad como el problema de la unidad de área modificable en dos sentidos: escala y zonificación. Un problema de escala se presenta cuando se obtienen resultados distintos con los mismos datos agrupados en unidades espaciales de tamaño cada vez mayor, como sería el caso de localidad-municipio-entidad. Y uno de zonificación ocurre cuando los resultados varían si los datos se organizan en unidades espaciales de forma distinta, aunque el número de unidades sea constante (Jelinski y Wu, 1996). De aquí la importancia de elegir una unidad de análisis espacial adecuada para representar la toma de decisiones, una elección que siempre está limitada por la disponibilidad de la información a escalas geográficas detalladas y que, por tanto, genera resultados que se deben interpretar cuidadosamente.

También hay que considerar que el comportamiento de las variables explicativas no es homogéneo sino diferenciado, a esto se le conoce como no estacionariedad espacial, y significa que la tenencia de la tierra, la densidad de población, la pendiente o la precipitación ejercen una influencia distinta sobre los procesos de CCUS, dependiendo del lugar que se analice, y para estudiarlo se han desarrollado modelos locales (ML). A diferencia de los modelos de regresión global, que calculan una ecuación para toda un área de estudio, los ML construyen ecuaciones locales con las variables explicativas y la dependiente, para cada unidad de análisis espacial: entidad federativa, municipio o localidad, y se ajustan agrupando observaciones contiguas a un punto de referencia. El supuesto principal es que las unidades espaciales más cercanas influyen más sobre el comportamiento en ese punto (Fotheringham, Brunsdon y Charlton, 2002; Pineda et al., 2010), una condición que resulta habitual en regiones especializadas en algún proceso productivo. Pineda et al. realizaron análisis de CCUS con ML, para comprender las causas de la pérdida forestal que actúan a escala global y local en el Estado de México, entre 1993 y el año 2000; con esta técnica obtuvieron mejores indicadores de ajuste estadístico en los ML que en los modelos de regresión global, y concluyeron que la relación entre las variables explicativas y la deforestación presenta un comportamiento espacial heterogéneo. Los resultados de algunas variables mostraron parámetros que indicaron una gran relación con la deforestación en ciertas zonas del área de estudio, pero que actuaron de forma contraria en otras zonas (2010, p. 583).

Además de los modelos locales, es posible utilizar otras formas para analizar los CCUS, entre las herramientas principales están el análisis de puntos calientes de deforestación (hot spots) (Li, Liu y Huang, 2017), las estadísticas de intensidad de cambio (Zhou, Huang, Pontius y Hong, 2014), índices de conectividad y fragmentación (Foltête, Girardet y Clauzel, 2014), así como otro tipo de índices de vegetación que se pueden analizar históricamente (Healey, Cohen, Zhiqiang y Krankina, 2005).

\section{Modelo de análisis CCUS-TAR}

Premisas para el vínculo teórico-metodológico desde la TAR

La TAR permite establecer varias premisas útiles para construir un vínculo teó-

rico-metodológico con el análisis de CCUS. La primera se refiere al principio de 
simetría, que coloca al sociólogo en una posición simétrica de observación sobre las asociaciones entre actores humanos y no humanos. Esto implica que la tierra, vista como sinónimo de biosfera tiene capacidad de interacción. Por lo tanto, la pendiente, la altitud, el tipo de suelo, las distancias a cuerpos de agua y las carreteras, entre otros elementos definidos espacialmente, son indispensables para explicar el proceso de traducción, porque representan entidades que participan de la acción.

Una consideración metodológica en este punto es que los actores humanos revelan con facilidad su papel como mediadores de la acción, mientras que los no humanos "pasan rápidamente de ser mediadores a ser intermediarios" (Latour, 2008, p. 117), por ello es necesario desarrollar estrategias para hacerlos visibles. El papel de los objetos como mediadores puede ser visible en las innovaciones, los accidentes, la investigación documental y mediante la ficción (Latour, 2008, p. 121). A esta lista es posible agregar los mapas, que constituyen una representación gráfica de relaciones espaciales.

La segunda premisa alude al papel que juegan los mediadores no humanos, como elementos que consolidan las relaciones de poder. Los roles de poder 0 subordinación, adquiridos por los humanos en los procesos de traducción, se afianzan a través de la multiplicidad de actores no humanos que participan. La estabilidad de la movilización de una red dependerá de la eficiencia de estos mediadores, de ahí la importancia de lograr que sean visibles. Una manera de conseguirlo es por medio del mapeo de las relaciones entre los actores de la red de manejo de tierras, por ejemplo cuando se representa la relación entre la distribución espacial de los recursos públicos y la tenencia de la tierra, o entre la de los suelos más aptos y los ingresos de la población rural.

En la tercera premisa se reconoce que las interacciones son locales, por lo tanto localizadas, esto significa que siempre es posible encontrar un vínculo con las construcciones estructurales, a través del seguimiento de las actividades locales. Para rastrear el origen de las decisiones sobre manejo, no es necesario sustituir la complejidad de conexiones que impulsan a los tomadores de decisiones con explicaciones resumidas en conceptos como fuerzas del mercado, prácticas culturales o globalización. Estos procesos complejos tienen representantes locales personificados en técnicos agropecuarios, funcionarios públicos, empresas financieras, distribuidores de insumos agropecuarios o bien en los objetos con los que interactúan, como semillas, maquinaria, suelos y agua, entre muchos. Una cuarta premisa plantea que las interacciones locales, que configuran la red que se teje en torno a las decisiones sobre manejo de tierras, están ancladas espacialmente. El fundamento básico de esta premisa es que la tierra es uno de los principales actores-red que participan en ella.

Premisas para el vínculo teórico-metodológico desde el análisis de CCUS

La primera premisa, desde el análisis espacial para trazar el vínculo con la TAR, es que los modelos espaciales de CCUS pueden representar las configuraciones espaciales, producto de las asociaciones entre actores-red. Esta afirmación se sustenta en que las interacciones entre los actores participantes de la red de manejo de tierras configuran la superficie terrestre y el CCUS registra la huella 
de éstas. Un modelo que relaciona los CCUS con la pendiente del terreno representa la forma en que los tomadores de decisiones son impulsados a actuar en respuesta a los obstáculos, o las facilidades que imponen las geoformas (lomeríos, valles y/o montañas) a sus actividades productivas. La segunda premisa confirma lo planteado, que las relaciones espaciales representan las de poder. La tercera admite el principio de no estacionariedad de los fenómenos espaciales porque, como se estableció en la tercera premisa desde la TAR, las interacciones son locales, por lo tanto localizadas. De aquí que los ML son un ejercicio adecuado para mostrar el comportamiento diferenciado de la toma de decisiones, en relación con las condiciones espaciales. Ciertas geoformas pueden ejercer mayor presión sobre la deforestación en unas zonas respecto a otras, o bien favorecerla en algunas e inhibirla en otras. Las estadísticas espaciales, producto de los ML, constituyen la representación numérica de la dimensión, fortaleza e incluso el sentido de estas relaciones localizadas. En este sentido, los mapas, producto de los ML, son una manera de utilizar la ficción, un recurso heurístico para facilitar la observación y la descripción de las interacciones locales, que se tejen en torno al manejo de tierras.

Tabla 1. Síntesis de las implicaciones ontológicas, epistemológicas y metodológicas que vinculan las premisas desde los enfoques TAR y CCUS

\begin{tabular}{|c|c|c|c|c|}
\hline Premisas TAR & $\begin{array}{l}\text { Principio de } \\
\text { simetría }\end{array}$ & $\begin{array}{l}\text { Los mediadores } \\
\text { no humanos } \\
\text { consolidan } \\
\text { las relaciones } \\
\text { de poder }\end{array}$ & $\begin{array}{c}\text { Las } \\
\text { interacciones } \\
\text { son locales, } \\
\text { por lo tanto } \\
\text { localizadas }\end{array}$ & $\begin{array}{l}\text { Las interacciones } \\
\text { locales están } \\
\text { ancladas } \\
\text { espacialmente }\end{array}$ \\
\hline \multirow{3}{*}{$\begin{array}{l}\text { Implicaciones } \\
\text { ontológicas }\end{array}$} & \multicolumn{4}{|c|}{ La tierra tiene capacidad de interacción } \\
\hline & \multicolumn{4}{|c|}{ La dimensión espacial es un atributo de los actores-red } \\
\hline & \multicolumn{4}{|c|}{$\begin{array}{c}\text { Las interacciones entre los actores participantes de la red de manejo } \\
\text { de tierras configuran la superficie terrestre }\end{array}$} \\
\hline $\begin{array}{l}\text { Implicaciones } \\
\text { epistemológicas }\end{array}$ & \multicolumn{4}{|c|}{ La dimensión espacial es un elemento explicativo } \\
\hline $\begin{array}{l}\text { Implicaciones } \\
\text { metodológicas }\end{array}$ & \multicolumn{4}{|c|}{ Los actores-red se pueden representar en variables espaciales } \\
\hline Premisas CCUS & $\begin{array}{l}\text { Los modelos } \\
\text { espaciales } \\
\text { de CCUS } \\
\text { representan las } \\
\text { configuraciones } \\
\text { producto } \\
\text { del manejo } \\
\text { de tierras }\end{array}$ & $\begin{array}{l}\text { Las relaciones } \\
\text { espaciales } \\
\text { pueden } \\
\text { representar } \\
\text { relaciones } \\
\text { de poder }\end{array}$ & $\begin{array}{c}\text { Principio } \\
\text { de no } \\
\text { estacionariedad. } \\
\text { Comportamiento } \\
\text { espacial } \\
\text { diferenciado } \\
\text { de los conductores } \\
\text { del cambio }\end{array}$ & $\begin{array}{c}\text { Los modelos } \\
\text { producto del } \\
\text { análisis espacial } \\
\text { aportarán hipótesis } \\
\text { acerca de las } \\
\text { decisiones sobre } \\
\text { manejo de tierras } \\
\text { para ser probadas } \\
\text { en el proceso } \\
\text { de traducción }\end{array}$ \\
\hline
\end{tabular}

Fuente: elaboración propia. 
En el análisis de CCUS, los datos erróneos o incompletos conducen a problemas de escala o zonificación. En donde los tomadores de decisiones no se encuentren bien representados en las variables espaciales, se podrían presentar problemas de la unidad de área modificable o de interpretaciones erróneas. Sin embargo, esto se puede mitigar si se considera una cuarta premisa: que los modelos producto del análisis espacial solo aportarán hipótesis restringidas a la escala temporal y espacial disponible, pero que pueden constituir el punto de partida para rastrear asociaciones espaciales en las interacciones locales. Estas hipótesis serán corroboradas o descartadas en la descripción del proceso de traducción porque, como se plantea en la cuarta premisa de la TAR, las interacciones que configuran la red que se teje en torno a las decisiones sobre manejo de tierras están ancladas espacialmente.

\section{Rastrear asociaciones espaciales en el manejo de tierras}

Desde las premisas planteadas se concluye que la dimensión espacial es el eje que vincula la TAR y el análisis de CCUS. Así, el objetivo del modelo construido aquí, y que vincula ambos enfoques, es rastrear las asociaciones espaciales producto de las redes que se tejen en torno al manejo de tierras, para comprender la toma de decisiones. El punto de partida de esta propuesta debe situar al investigador en la controversia sobre el CAG, que documenta tres cosas: a) la ocurrencia de cambios importantes en la tierra; b) la probada responsabilidad de los humanos sobre esos cambios y c) que la respuesta de la tierra ante ellos está imponiendo límites al desarrollo de la actividad humana, centrada en el modelo de acumulación de capital dominante.

El abordaje metodológico propuesto se compone de tres fases, la primera se guía por los argumentos de la controversia, cuyo objetivo es describir patrones de CCUS, e identificar relaciones espaciales entre esos cambios e indicadores de las actividades humanas. En este punto de partida, los mapas de cambio y modelos de CCUS constituyen la herramienta básica para representar las asociaciones espaciales entre los actores que integran la red de manejo de tierras. Para fortalecer este ejercicio se pueden aprovechar otras herramientas del análisis espacial como la identificación de puntos calientes, las estadísticas de intensidad de cambio y el cálculo de diversos índices de conectividad, fragmentación y vegetación. La elaboración e interpretación de estos mapas y estadísticas espaciales se debe hacer partiendo de las cuatro premisas establecidas para el análisis de CCUS (ver Figura 1). Por lo tanto, el primer resultado de esta fase es una serie de hipótesis generadas a partir del análisis espacial de los CCUS sobre las relaciones entre actores-red; el segundo es la identificación de una ventana local, donde se registren cambios extremos de recuperación o pérdida de cubiertas con el fin de profundizar en la siguiente fase y probar las hipótesis resultantes.

La segunda fase pretende describir el proceso de traducción que generó las configuraciones espaciales observadas en los mapas; consiste en rastrear la cadena de mediadores humanos y no humanos, que impulsan a los tomadores de decisiones en una ventana local; describir las líneas de la estrella que mantienen ancladas localmente estas relaciones espaciales e identificar si trasportan auto- 
Figura 1. Modelo de análisis CCUS-TAR, para rastrear asociaciones espaciales en el manejo de tierras

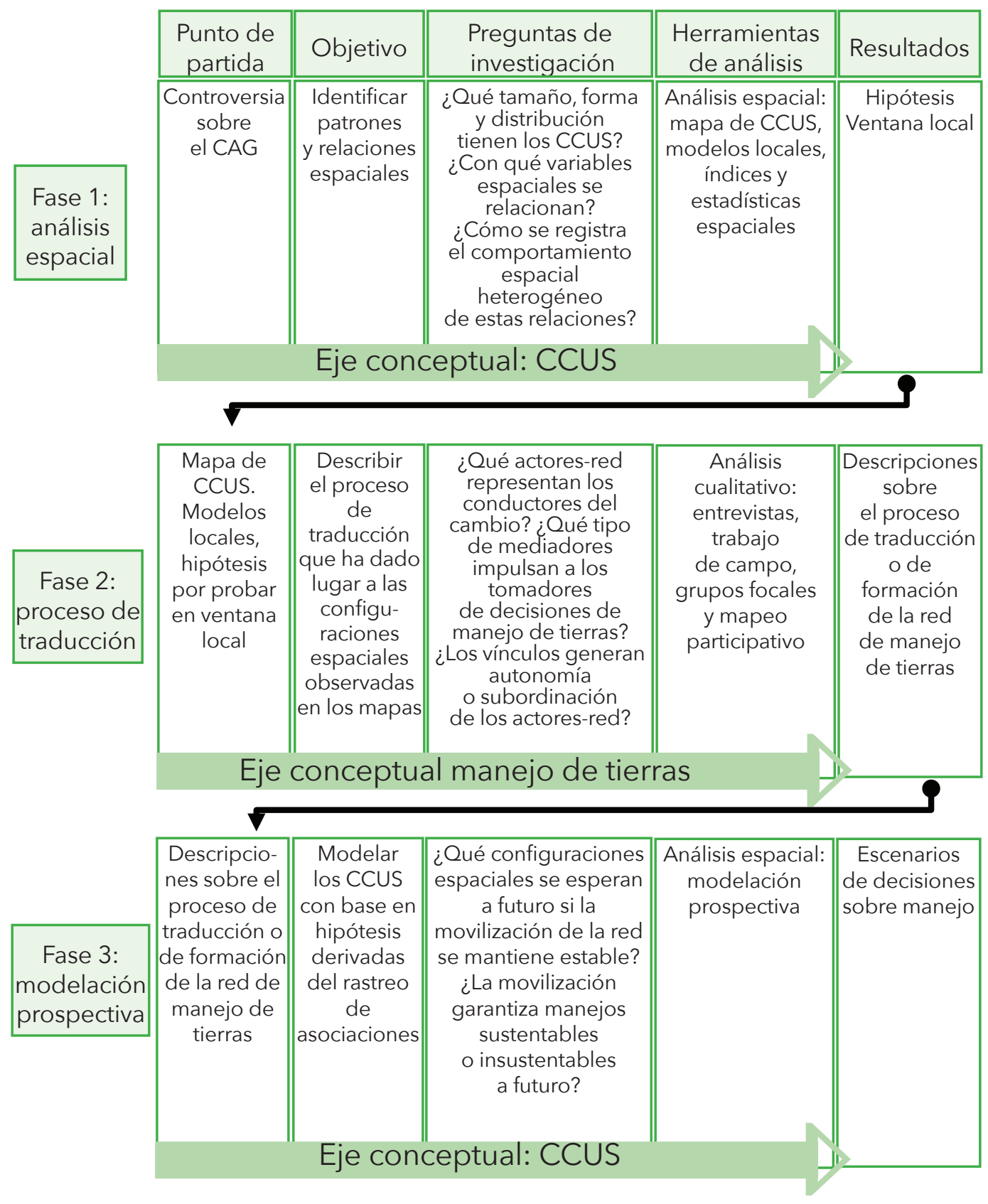

Fuente: elaboración propia. 
nomía o subordinación. El trabajo de rastreo de las asociaciones espaciales en esta fase se realiza con base en las premisas planteadas desde la TAR (ver Figura 1 ), y requiere las herramientas de investigación cualitativa: entrevistas, recorridos de campo, trayectorias de vida, grupos focales y mapeo participativo, que ofrecen la posibilidad de recrear y reflexionar con los tomadores de decisiones sobre la relación histórica establecida con sus tierras, a través del uso de imágenes satelitales y ejercicios de cartografía social (Braceras, 2012). El resultado es la descripción de las cadenas de mediaciones entre humanos y no humanos, que contendrán información sobre los roles que juegan los actores-red en el manejo de tierras; la identificación de los mediadores clave, que garantizan la estabilidad en la movilización de la red y la explicación, desde la perspectiva de los actores, de las razones que condujeron las decisiones sobre el manejo de tierras en la ventana local.

Una red estable reproduce los manejos sustentables o insustentables de la tierra. Por esta razón, el conocimiento sobre estos procesos de traducción es el punto de partida para la tercera fase, donde se retoma al análisis espacial para desarrollar ejercicios de modelación prospectiva del CCUS, con base en hipótesis derivadas de los resultados del rastreo de asociaciones. Estos modelos ya no son construcciones estadísticas elaboradas, con sustento teórico débil, sino producto de las asociaciones espaciales que los actores van desplegando en la explicación de sus motivos para la acción.

Los mapas, producto de esta última fase, podrían ser reconceptualizados como escenarios de decisiones sobre manejo de tierras; éstos contienen información acerca de los efectos de la actividad humana sobre la superficie terrestre, pero también representarán la reorganización espacial que los cambios ambientales generan sobre las actividades humanas. En este sentido, pueden ser de gran utilidad para responder preguntas futuras sobre los CCUS, y servir como insumos para los actores que participan en la red promoviendo la reflexión sobre iniciativas que conduzcan a manejos sustentables.

\section{Conclusiones}

Con la revisión y el modelo de análisis expuestos aquí se propuso resolver algunas limitaciones ontológicas y, por ende, epistemológicas que presentan los enfoques examinados en el abordaje del estudio de las decisiones sobre el manejo de tierras, y reconocer la capacidad de interacción de la tierra o la naturaleza, entendida como un actor-red (biosfera), así como integrar teórica y metodológicamente la dimensión espacial. Asimismo, se planteó superar algunas de las debilidades de los enfoques de análisis de CCUS en la integración de los componentes sociales, para la explicación de los cambios de uso de suelos.

La ventaja principal de esta propuesta de investigación sobre otros ejercicios es que las estadísticas espaciales no son datos vacíos sino representaciones del comportamiento de actores-red, en su tarea de establecer asociaciones espaciales o bien interacciones locales. En la primera fase, las estadísticas espaciales no explican el comportamiento de los conductores del cambio, solo ofrecen hipótesis sobre relaciones espaciales que se deben rastrear en las interacciones 
locales de los actores-red. Los mapas dejan de ser un fin, y se convierten en un medio para la investigación sobre las decisiones de manejo de tierras. En la tercera fase, los mapas de nuevo constituyen un fin, aunque las hipótesis que los alimentan están basadas en argumentos directos del actor-red, en explicaciones proporcionadas por los tomadores de decisiones.

La perspectiva de la TAR también se enriquece en este ejercicio. La integración explícita de la dimensión espacial en el mapeo de las interacciones locales entre actores-red fortalece el enfoque, tanto en el punto de partida de la primera fase, para explorar las controversias sobre el manejo de tierras, como en la última, donde se propone traducir los resultados del rastreo de asociaciones espaciales en hipótesis sobre las configuraciones espaciales a futuro, para alimentar escenarios de decisiones sobre manejo.

Sin duda, la controversia actual sobre el CAG produce propuestas alternativas abundantes para superar los vacíos teórico-metodológicos en la explicación de la toma de decisiones sobre el manejo de tierras y sus consecuencias ambientales. Sin embargo, en este artículo se sostiene que el reconocimiento de la capacidad de interacción de la tierra y sus componentes en el desarrollo de esta controversia resulta un primer paso, esencial para transitar de los modelos explicativos a los trasformadores de la realidad socionatural, de tal manera que los tomadores de decisiones se desenvuelvan en redes sustentables y justas para todos los participantes, en donde se rompan los vínculos con los actores de conductas proclives a consolidar la maximización de la ganancia como el fin supremo del manejo de tierras, y se construyan nuevos, con entidades mediadoras promotoras de racionalidades que reintegren la vida humana a la naturaleza, como proponía Polanyi (2007).

En suma, el modelo expuesto ofrece posibilidades para la trasformación socioambiental en varios sentidos: a) como instrumento de diagnóstico y visibilización de la red de mediadores humanos y no humanos, que amplía o limita la toma de decisiones sobre el manejo de tierras exhibiendo los vínculos y actores que conducen a manejos insustentables; b) como instrumento de generación y difusión de información, que favorezca la identificación y la vinculación con actores impulsores de conductas pro ambientalistas en la red y c) como proveedor de insumos que respalden el desarrollo de iniciativas que contrarresten el CAG, como políticas públicas, leyes que castiguen las conductas extractivistas, contaminantes y destructivas de la tierra; la movilización social y acciones colectivas, que sancionen estas conductas en los mercados convencionales, o se orienten a la creación de otros alternativos o de modelos de producción que también lo sean, como la agricultura cívica (Salgado, 2015), y otras iniciativas que se beneficien de la investigación sobre el CAG, para emigrar de un paradigma de adaptación hacia otro de trasformación (O’Brien, 2012a, 2012b).

\section{Agradecimientos}

Los autores agradecen al Fondo Sectorial del Consejo Nacional de Ciencia y Tecnología-Instituto Nacional de Estadística y Geografía el financiamiento recibido, en el marco de su convocatoria 2013-1, para redactar este artículo, que forma 
parte de los resultados del proyecto "Análisis espacio-temporal de la vulnerabilidad del paisaje utilizando percepción remota y métodos espaciales: un estudio interdisciplinario y multiescalar en cuatro regiones del país", clave 210080.

\section{Referencias}

Aranda, J. M. (2004). Principales desarrollos de la sociología ambiental. Ciencia Ergo Sum, 11(2), 199-208. Recuperado de http://www.redalyc.org/articulo. oa?id=10411210

Berkes, F., Colding, J. y Folke, C. (2003). Navigating social-ecological systems: Building resilience for complexity and change. Cambridge: Cambridge University Press. Recuperado de http://assets.cambridge.org/052181/5924/ sample/0521815924ws.pdf

Banco Mundial. (2008). Manejo sostenible de la tierra: desafíos, posibilidades y costos de oportunidad. Colombia: Banco Mundial y Mayol Ediciones S. A. Recuperado de http://documentos.bancomundial.org/curated/ es/901001468170041631/Manejo-sostenible-de-la-tierra-desafios-posibilidades-y-costos-de-oportunidad

Bourdieu, P. (1999). La miseria del mundo. Madrid: Fondo de Cultura Económica de Argentina, S. A.

Bourdieu, P. y Wacquant, L. (2005). Una invitación a la sociología reflexiva. Buenos Aires: Siglo XXI Editores.

Braceras, I. (2012). Cartografía participativa: herramienta de empoderamiento y participación por el derecho al territorio (tesis de maestría). Recuperada de https://geoactivismo.org/wp-content/uploads/2014/10/Tesina_n_2_Iratxe_Braceras.pdf

Castree, N. (2016). Geography and the new social contract for global change research. Transactions of the Institute of British Geographers, (41), 328-347. doi: $10.1111 / \operatorname{tran} .12125$

Chowdhury, R. R. (2006). Driving forces of tropical deforestation: The role of remote sensing and spatial models. Singapore Journal of Tropical Geography, (27), 82-101. doi: 10.1111/j.1467-9493.2006.00241.x

Consejo Latinoamericano de Ciencias Sociales (CLACSO). (2018). Cambio ambiental global. Grupo de Trabajo sobre Cambio Medioambiental Global, Territorio y Políticas Ambientales. Recuperado de https://clacsoambientalglobal. wordpress.com/cambio-ambiental-global-2/

Cote, M. y Nightingale, A. J. (2012). Resilience thinking meets social theory: Situating social change in socio-ecological systems (SES) research. Progress in Human Geography, 36(4), 475-489. doi: 10.1177/0309132511425708

DellaSala, D. A., Goldstein, M. I., Elias, S., Jennings, B., Lacher, T. E., Mineau, P. y Pyare. S. (2018). The anthropocene: How the great acceleration is transforming the planet at unprecedented levels. En D. A. DellaSala y M. I. Goldstein (eds.), Encyclopedia of the anthropocene (pp. 1-7). Oxford: Elsevier, Book Aid International. doi:10.1016/B978-0-12-809665-9.09957-2

Eichholz, M. , Assche, K. V. , Oberkircher, L. yHornidge, A. K. (2012). Trading capitals? Bourdieu, land and water in rural Uzbekistan. Journal of Environmental Planning and Management, 56(6), 868-892. doi: 10.1080/09640568.2012.708650 
Entwisle, B., Walsh, S. J., Rindfuss, R. R. y Chamratrithirong, A. (1998). Landuse/land-cover and population dynamics, Nang Rong, Thailand. En D. Liverman, E. F. Moran, R. R. Rindfuss y P. C. Stern (eds.), People and pixels. Linking remote sensing and social science (pp. 121-144). Washington: National Academy Press.

Food and Agriculture Organization (FAO). (2016). Portal de suelos de la FAO. Recuperado de http://www.fao.org/soils-portal/soil-management/es/

Foltête, J. C., Girardet, X. y Clauzel, C. (2014). A methodological framework for the use of landscape graphs in land-use planning. Landscape and Urban Planning, (124), 140-150. doi: 10.1016/j.landurbplan.2013.12.012

Fotheringham, A. S., Brunsdon, C. y Charlton, M. (2002). Geographically weighted regression. The analysis of spatially varying relationships. Newcastle: John Wiley and Sons.

Geist, H. y Lambin, E. (2002). Proximate causes and underlying driving forces of tropical deforestation. BioScience, 52(2), 143-150. doi: 10.1641/0006-3568(2002)052[0143:PCAUDF]2.0.CO;2

Geoghegan, J., Cortina, S., Klepeis, P., Mendoza, P. M., Ogneva, Y., Chowdhury, R., Turner II, B. L. y Vance, C. (2001). Modeling tropical deforestation in the Southern Yucatán peninsular region: Comparing survey and satellite data. Agriculture, Ecosystems and Environment, (85), 25-46. doi: 10.1016/S01678809(01)00201-8

Giddens, A. (1984). The constitution of society. Outline of the theory of structuration. Oxford: Polity Press Editorial.

Giddens, A. (2014). Sociología. Madrid: Alianza Editorial.

Goodman, D. (1999). Agro-food studies in the 'age of ecology': Nature, corporeality, bio-politics. Sociologia Ruralis, 39(1), 17-38. doi: 10.1111/14679523.00091

Gray, B. J. y Gibson, J. W. (2013). Actor-networks, farmer decisions, and identity. Culture, Agriculture, Food and Environment, 35(2), 82-101. doi: 10.1111/ cuag. 12013

Hansen, M. C., Potapov, P. V., Moore, R., Hancher, M., Turubanova, S. A., Tyukavina, A. ... Townshend, J. R. G. (2013). High-resolution global maps of 21st-century forest cover change. Science, (342), 850-853. doi: 10.1126/ science. 1244693

Healey, S. P., Cohen, W. B., Zhiqiang, Y. y Krankina, O. N. (2005). Comparison of tasseled cap-based landsat data structures for use in forest disturbance detection. Remote Sensing of Environment, (97), 301-310. doi: 10.1016/j. rse.2005.05.009

Huggett, R. J. (1999). Ecosphere, biosphere, or gaia? What to call the global ecosystem. Global Ecology and Biogeography, (8), 425-431. doi: 10.1046/j.1365-2699.1999.00158.x

Panel Intergubernamental del Cambio Climático (IPCC). (2013). Resumen para responsables de políticas. En T. F. Stocker, D. Qin, G-K Plattner, M. M. B Tignor, S. K. Allen, J. Boschung, A. Nauels, Yu Xia, V. Bex y P. M. Midgley (eds.), Cambio climático 2013: bases físicas. Contribución del grupo de trabajo I al Quinto Informe de Evaluación del Grupo Intergubernamental de Expertos sobre el Cambio Climático (pp. 3-30). Cambridge y Nueva York: Cambridge Uni- 
versity Press. Recuperado de https://www.ipcc.ch/pdf/assessment-report/ ar5/wg1/WG1AR5_SPM_brochure_es.pdf

Panel Intergubernamental del Cambio Climático (IPCC). (2014). Technical Summary. En O. Edenhofer, R. Pichs-Madruga, Y. Sokona, E. Farahani, S. Kadner, K. Seyboth, A. Adler, I. Baum, S. Brunner, P. Eickemeier, B. Kriemann, J. Savolainen, S. Schlömer, C. von Stechow, T. Zwickel y J. C. Minx (eds.), Climate change 2014: mitigation of climate change. Contribution of Working Group III to the Fifth Assessment Report of the Intergovernmental Panel on Climate Change (pp. 33-108) Cambridge y Nueva York: Cambridge University Press. Recuperado de https://www.ipcc.ch/pdf/assessment-report/ar5/wg3/ipcc_ wg3_ar5_frontmatter.pdf

Jelinski, D. E. y Wu, J. (1996). The modifiable areal unit problem and implications for landscape ecology. Landscape Ecology, 11(3), 129-140. doi: 10.1007/ BF02447512

Lambin E. F., Geist, H. J. y Lepers, E. (2003). Dynamics of land-use and land-cover change in tropical regions. Annual Review of Environment and Resources, (28), 205-241. doi: 10.1146/annurev.energy.28.050302.105459

Latour, B. (2008). Reensamblar lo social: una introducción a la teoría del actor-red. Buenos Aires: Manantial.

Leichenko, R. y Eisenhauer, D. C. (2017). Global environmental change: Human dimensions. En D. Richardson, N. Castree, M. F. Goodchild, A. Kobayashi, W. Liu, y R. A. Marston (eds.), The international encyclopedia of geography (pp. 1-11). John Wiley \& Sons, Ltd. doi: 10.1002/9781118786352.wbieg0649

Li, Y., Liu, G. y Huang, C. (2017). Dynamic changes analysis and hotspots detection of land use in the central core functional area of Jing-Jin-Ji from 2000 to 2015 based on remote sensing data. Mathematical Problems in Engineering, (2017), 1-16. doi: 10.1155/2017/2183585

Mariñez, F. (2005). Funcionalismo y reconfiguración social. Una revisión crítica de la sociología de Talcott Parsons. Trayectorias, (19), 32-45. Recuperado de http: / / trayectorias.uanl.mx/19/funcionalismo.html

Mastrangelo, A. V. (2009). Análisis del concepto de recursos naturales en dos estudios de caso en Argentina. Ambiente \& Sociedad, 12(2), 341-355. doi: 10.1590/S1414-753X2009000200009

Mendiola, I. (2012). Habitando espacios socionaturales: reflexiones desde la ecología política. En F. Tirado y D. López (eds.), Teoría del actor-red. Más allá de los estudios de ciencia y tecnología (pp. 243-283). Barcelona: Amentia Editorial.

Meyer, W. B. y Turner II, B. L. (1992). Human population growth and global landuse/cover change. Annual Review of Ecology and Systematics, (23), 39-61. doi: 10.1146/annurev.es.23.110192.000351

Moreno, F. A. (1988). El lugar de lo rural. En J. Zepeda (coord.), Las sociedades rurales hoy (pp. 113-126). Zamora: El Colegio de Michoacán.

National Research Council (NRC). (2013). Advancing land change modeling: Opportunities and research requirements. Washington: The National Academies Press, National Research Council. Recuperado de http://www.nap.edu/ catalog.php?record_id=18385 
O’Brien, K. (2012a). Global environmental change II: from adaptation to deliberate transformation. Progress in Human Geography, 36(5), 667-676. doi: 10.1177/0309132511425767

O'Brien, K. (2012b). Global environmental change III: closing the gap between knowledge and action. Progress in Human Geography, 37(4), 587-596. doi: 10.1177/0309132512469589

Overmars, K. P. y Verburg, P. H. (2005). Analysis of land use drivers at the watershed and household level: Linking two paradigms at the Philippine forest fringe. International Journal of Geographical Information Science, 19(2), 125-152. doi: 10.1080/13658810410001713380

Pineda, N. B., Bosque, J., Gómez, M. y Franco, R. (2010). Exploring the driving forces behind deforestation in the State of Mexico (Mexico) using geographically weighted regression. Applied Geography, (30), 576-591. doi: 10.1016/j. apgeog.2010.05.004

Polanyi, K. (2007). La gran transformación. Crítica del liberalismo económico (primera edición 1947). Argentina: Quipu Editorial.

Raedeke, A. H. y Rikoon, J. S. (1997). Temporal and spatial dimensions of knowledge: Implications for sustainable agriculture. Agriculture and Human Values, (14), 145-158. doi: 10.1023/A:1007346929150

Rosales, M. R. (2006). Modernidad, naturaleza y riesgo. En P. L. Sotolongo y C. J. Delgado (coords.), La revolución contemporánea del saber y la complejidad social. Hacia unas ciencias sociales de nuevo tipo (pp. 213-221). Buenos Aires: Consejo Latinoamericano de Ciencias Sociales. Recuperado de http://bibliotecavirtual.clacso.org.ar/ar/libros/campus/soto/soto.html

Salgado, R. (2015). Agricultura sustentable y sus posibilidades en relación con consumidores urbanos. Estudios Sociales, (45), 114-140. Recuperado de https://www.ciad.mx/estudiosociales/index.php/es/article/view/184/105

Serneels, S. y Lambin, E. F. (2001). Proximate causes of land-use change in Narok District, Kenya: A spatial statistical model. Agriculture, Ecosystems and Environment, (85), 65-81. doi: 10.1016/S0167-8809(01)00188-8

Smelser, N. y Swedberg, R. (2005). Introducing economic sociology. En N. Smelser y R. Swedberg (eds.), The handbook of economic sociology (pp. 3-25). Nueva York: Princeton University Press.

Soares-Filho, B., Alencar, A., Nepstad, D., Cerqueira, G., Vera, M. C., Rivero, S., Solorzano, L. y Voll, E. (2004). Simulating the response of land-cover changes to road paving and governance along a major Amazon highway: The SantarémCuiabá corridor. Global Change Biology, (10), 745-764. doi: 10.1111/j.15298817.2003.00769.x

Valembois, V. (1981). El binomio hombre-naturaleza en el indio mesoamericano. Revista de Filosofía, (49-50), 115-120. Recuperado de http://www.inif. ucr.ac.cr/recursos/docs/Revista\%20de\%20Filosof\%C3\%ADa\%20UCR/Vol.\%20 XIX/49-50/El\%20binomio\%20Hombre-Naturaleza\%20en\%20el\%20indio\%20mesoamericano.pdf

Vance, C. y Geoghegan, J. (2002). Temporal and spatial modelling of tropical deforestation: A survival analysis linking satellite and household survey data. Agricultural Economics, (27), 317-332. doi: 10.1016/S0169-5150(02)00074-9 
Venturini, T. (2010). Diving in magma: How to explore controversies with actor-network theory. Public Understanding of Science, 19(3), 258-273. doi: 10.1177/0963662509102694

Williams, D. R. y Patterson, M. E. (1996). Environmental meaning and ecosystem management: Perspectives from environmental psychology and human geography. Society \& Natural Resources, 9(5), 507-521. doi: 10.1080/08941929609380990

Young, O. R., Berkhout, F., Gallopin, G. C., Janssen, M. A., Ostrom, E. y Leeuw, S. (2006). The globalization of socio-ecological systems: An agenda for scientific research. Global Environmental Change, (16), 304-316. doi: 10.1016/j. gloenvcha.2006.03.004

Zhou, P., Huang, J., Pontius Jr., R. G. y Hong, H. (2014). Land classification and change intensity analysis in a coastal eatershed of Southeast China. Sensors, (14), 11640-11658. doi: 10.3390/s140711640 